\title{
Analysis of the Dielectric Loss in the Transmission Line Based on Time Domain BLT Equation
}

\author{
Wanjin Wang \\ Section 94, PLA 91550 \\ Dalian, China \\ wwj.1982-2002@163.com
}

\author{
Zhiwu Xuan \\ Section 94, PLA 91550 \\ Dalian, China \\ xuanzw@126.com
}

\begin{abstract}
Dielectric loss is caused due to imperfect dielectric insulation, in order to study the impact of the dielectric loss consider a uniform loss transmission line with leak conductance. The BLT equation from the frequency domain to time domain is derived to improve the time domain BLT equation, and the aim of using the time domain BLT equation to calculate load voltage of the transmission line with transient signal source, through the calculation results to analyze the impact of the dielectric loss. The results showed that the attenuation of the terminal load transient response voltage occurred when the dielectric loss exists, and this effect is nonlinear.
\end{abstract}

Keywords-Dielectric Loss;Leak Conductance;Coaxial Cable;

BLT Equation

\section{INTRODUCTION}

When the high frequency current through the transmission line, since the insulating imperfect and there is leakage current between conductors, i.e. along the entire distribution of the leakage conductance. Usually transient signal transmission line are assumed dielectric insulating properties is the ideal medium, which can simplify the research will undoubtedly increase the possibility of error, but guidance on practical problems. In the premise of the assumption was no dielectric loss, [1] used in the BLT equation of the time-domain response voltage of the terminal of the coaxial transmission line were calculated Although it is possible to simplify the BLT equation derivation process of the time domain and the convenience of calculation, but not considering the dielectric loss which spread high frequency signal may be generated situation, the timedomain BLT equation is still not perfect in theory, the lack of objective theoretical basis in theory to guide practice. This article [1] when the domain BLT equation conduct the improved, considering arising from medium leakage drain conduction of the case, and based on the transmission line terminal response voltage under no dielectric electrical loss and dielectric loss situation carried the study, not only to improve the BLT equation in the time domain, dielectric loss voltage transmission line terminal load transient response.

\section{TRANSMISSION LINE TERMINAL RESPONSE}

In the case of assuming loss, Uniform transmission line can be used unit length distribution resistance $R^{\prime}$, inductance
$L^{\prime}$ capacitor $C^{\prime}$ of the leakage conductance $G^{\prime}$ and $\sqrt{s}$ as the ratio of loss to describe skin, For homogeneous loss transmission line, the series impedance and shunt admittance per unit length in the frequency domain in the form of [1]

$$
Z^{\prime}=s L^{\prime}+R^{\prime}+\xi \sqrt{s} \text { and } Y^{\prime}=s C^{\prime}+G^{\prime}
$$

For the inner and outer radii of the $a$ and $b$ of the coaxial transmission line, the parameter $\xi$

$$
\xi=(1 / 2 \pi) \sqrt{\mu_{0} / b}(1 / a+1 / b)
$$

The propagation constant of the transmission line can be obtained by the above parameters as

$$
\gamma(s)=\sqrt{\left(s L^{\prime}+R^{\prime}+\xi \sqrt{s}\right)\left(s C^{\prime}+G^{\prime}\right)}
$$

Characteristic impedance

$$
Z_{c}(s)=\sqrt{\left(s L^{\prime}+R^{\prime}+\xi \sqrt{s}\right) /\left(s C^{\prime}+G^{\prime}\right)}
$$

Here the Laplace transform variable $s=\sigma+j \omega$ to define the complex frequency.

\section{A. transient propagation function}

In the case of low-loss and high-frequency $\left|R^{\prime} / s L^{\prime}\right| \ll 1,\left|\xi / s L^{\prime}\right| \ll 1,\left|G^{\prime} / s C^{\prime}\right| \ll 1$, The square root in the propagation constant of (2) can be approximated as

$$
\gamma(s) \approx s / v+a_{1} \sqrt{s}+a_{2}+a_{3}
$$

where the following definitions are used:

$$
v=1 / \sqrt{L^{\prime} C^{\prime}}, a_{1}=(\xi / 2) \sqrt{C^{\prime} / L^{\prime}}, a_{2}=\left(R^{\prime} / 2\right) \sqrt{C^{\prime} / L^{\prime}}, a_{3}=\left(G^{\prime} / 2\right) \sqrt{C^{\prime} / L^{\prime}}
$$

the spectral wave propagation term is

$$
G(s ; x)=e^{-\gamma(s) x} \approx e^{-s x / v} e^{-\sqrt{s} a_{1} x} e^{-a_{2} x} e^{-a_{3} x}
$$

using the Laplace transform, we calculate the transient propagation function of (5) for $t>x / v$ as

$$
g(t ; x)=e^{-a_{2} x} e^{-a_{3} x} e^{\left(-\left(a_{1} x\right)^{2} / 4(t-x / v)\right)} a_{1} x /\left(2 \sqrt{\pi(t-x / v)^{3}}\right)
$$

$(t>x / v)$

B. Surge Impedance and Admittance:

In the case of low-loss and high-frequency $\left|R^{\prime} / s L^{\prime}\right| \ll 1$, $\left|\xi / s L^{\prime}\right| \ll 1,\left|G^{\prime}\right| s C^{\prime} \mid \ll 1$ the frequency-domain characteristic 
impedance of (3) may also be approximated as

$$
Z_{c}(s) \approx \hat{Z}_{c}\left(1+R^{\prime} / 2 s L^{\prime+}+\xi / 2 \sqrt{s L^{\prime}}+G^{\prime} / 2 s C^{\prime}\right)
$$

where $\hat{Z}_{c}=\sqrt{L^{\prime} / C^{\prime}}$ is the high-frequency limit of the characteristic impedance of the line. Using the Laplace transform, the transient impedance function of (7) is

$$
Z_{c}(t) \approx \hat{Z}_{c} \delta(t)+\hat{Z}_{c} R^{\prime} / 2 L^{\prime} \Phi(t)+\hat{Z}_{c} \xi / 2 L^{\prime} \sqrt{\pi t}+\hat{Z}_{c} G^{\prime} / 2 C^{\prime} \Phi(t)
$$

A similar procedure can be used to transform the characteristic admittance of the line into the surge admittance $y_{c}(t)$.

The result is

$$
y_{c}(t) \approx \hat{Y} \delta(t)-\hat{Y}_{c} R^{\prime} \Phi(t) / 2 L^{\prime}-\hat{Y}_{c} \xi / 2 L^{\prime} \sqrt{\pi t}-\hat{Y}_{c} G^{\prime} \Phi(t) / 2 C^{\prime}
$$

where $\hat{Y}_{c}=\hat{Z}_{c}^{-1}=\sqrt{C^{\prime} / L^{\prime}}, \delta(t)$ is the Unit impulse function,

$$
\Phi(t) \text { is the unit-step function. }
$$

\section{Load Reflection Coefficients:}

The transient reflection coefficients (one for each end of the line) are different from the previous line parameters as they depend both on the line properties and on the nature of the termination impedances. The voltage reflection coefficient in the spectral domain for a resistive load is approximated as

$$
\rho(s)=\frac{R_{L}-Z_{c}(s)}{R_{L}+Z_{c}(s)} \approx \frac{R_{L}-\hat{Z}_{c}\left(1+R^{\prime} / 2 s L^{\prime}+\xi / 2 \sqrt{s} L^{\prime}+G^{\prime} / 2 s C^{\prime}\right)}{R_{L}+\hat{Z}_{c}\left(1+R^{\prime} / 2 s L^{\prime}+\xi / 2 \sqrt{s} L^{\prime}+G^{\prime} / 2 s C^{\prime}\right)}
$$

where $R_{L}$ is a terminal impedance of the load, and is assumed to be linear. Using the Laplace transform, we obtain the spectral representation for the reflection coefficient of (10)as

$$
r(t)=b_{1} / b_{2} \delta(t)+b_{8}\left(1 / \sqrt{\pi t}-\zeta_{1} e^{\zeta_{1}^{2} t} \operatorname{erfc}\left(\zeta_{1} \sqrt{t}\right)\right)-b_{9}\left(1 / \sqrt{\pi t}-\zeta_{2} e^{\zeta_{2}^{2} t} \operatorname{erfc}\left(\zeta_{2} \sqrt{t}\right)\right)
$$

Where a number of new parameters are defined as

$$
\begin{gathered}
b_{1}=R_{L}-\hat{Z}, \quad b_{2}=R_{L}+\hat{Z}, \quad b_{3}=\hat{Z}_{c} \frac{R^{\prime}}{2 L^{\prime}} \\
b_{4}=\hat{Z}_{c} \frac{\xi}{2 L^{\prime}}, \quad b_{5}=\hat{Z}_{c} \frac{G^{\prime}}{2 C^{\prime}}, \quad b_{6}=\frac{b_{4}}{b_{2}^{2}}\left(b_{1}+b_{2}\right) \\
b_{7}=\frac{b_{3}+b_{5}}{b_{2}^{2}}\left(b_{1}+b_{2}\right), \quad b_{8}=\frac{b_{7}}{\zeta_{2}-\zeta_{1}}\left(\zeta_{1}+\frac{b_{6}}{b_{7}}\right), \quad b_{9}=\frac{b_{7}}{\zeta_{2}-\zeta_{1}}\left(\zeta_{2}+\frac{b_{6}}{b_{7}}\right) \\
\zeta_{1,2}=\left(-b_{4} \pm \sqrt{b_{4}^{2}-4 b_{2}\left(b_{3}+b_{5}\right)} / 2 b_{2}, \quad \operatorname{erfc}(x)=2 \int_{x}^{\infty} e^{-t^{2}} d t / \sqrt{\pi}\right.
\end{gathered}
$$

\section{Application to the Time-Domain BLT Equation}

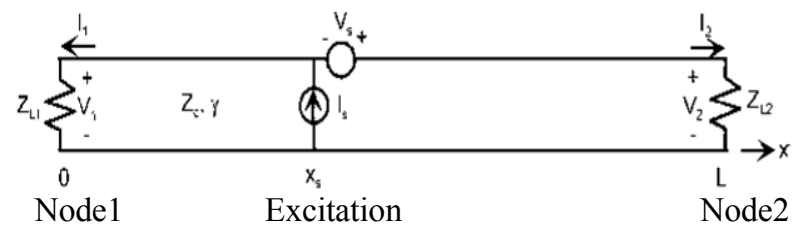

Figure 1. Simple transmission-line representation of the coaxial line

A transient BLT equation of the double-conductor transmission line shown in Figure 1 as

$\left[\begin{array}{l}v_{1}(t) \\ v_{2}(t)\end{array}\right]=\left[\begin{array}{cc}\delta(t)+r_{1}(t) & 0 \\ 0 & \delta(t)+r_{2}(t)\end{array}\right] *\left[\begin{array}{cc}m_{1}(t) & m_{2}(t) \\ m_{1}(t) & m_{2}(t)\end{array}\right] *\left[\begin{array}{l}s_{1}(t) \\ s_{2}(t)\end{array}\right]$

where the symbol $*$ denotes the time-domain convolution operator, and

$$
\begin{aligned}
r z_{1}(t) & =g(t ; L) * r_{1}(t) \\
r z_{2}(t) & =g(t ; L) * r_{2}(t) \\
m_{0}(t) & \equiv r z_{1}(t) * r z_{2}(t) \\
m(t)=\delta(t)+ & m_{0}(t) *\left\{\delta(t)+m_{0}(t) *\left[\delta(t)+m_{0}(t) *(\cdots)\right]\right\} \\
m_{1}(t) & =r z_{1}(t) * m(t) \\
m_{2}(t) & =r z_{2}(t) * m(t)
\end{aligned}
$$

Figure 1 here we use a lumped source and the transient source vector being expressed as

$\left[\begin{array}{l}s_{1}(t) \\ s_{2}(t)\end{array}\right]=\left[\begin{array}{l}-(1 / 2)\left(v_{s}(t)-z_{c}(t) * i_{s}(t)\right) * g\left(t ; x_{s}\right) \\ (1 / 2)\left(v_{s}(t)+z_{c}(t) * i_{s}(t)\right) * g\left(t ; L-x_{s}\right)\end{array}\right]$

Note that the source vector in (13) involves the transient propagation function $g(t ; x)$ of $(6)$ and uses line propagation distances of $X_{s}$ and $L-X_{s}$, which are the distances that the propagating waves must travel from the source at location $x=X_{s}$ to either end of the line.

At this point of the analysis, the matrix form of the transient-BLT equation (13) is not particularly beneficial for the evaluation of the load voltages $v_{1}(t)$ and $v_{2}(t)$. As a consequence, we can symbolically solve for these voltages to get the following two transient voltage equations to evaluate: $v_{1}(t)=\left(\delta(t)+r_{1}(t)\right) * m(t) *\left[s_{1}(t)+r z_{2}(t) * s_{2}(t)\right]$ 
$v_{2}(t)=\left(\delta(t)+r_{2}(t)\right) * m(t) *\left[r z_{1}(t) * s_{1}(t)+s_{2}(t)\right]$

\section{NUMERICAL EXAMPLE}

Here we filled to the inner and outer conductors' media uniformity of coaxial transmission line, for example, by calculating its signal source in a transient excitation terminal response to the load voltage to analyze the impact of the dielectric loss. With reference to Fig. 2, we assume the following parameters for the coaxial line: $a=2.5 \mathrm{~mm} ; \mathrm{b}$ $=9.345 \mathrm{~mm} ; \mathrm{c}=9.945 \mathrm{~mm} ; \Delta=\mathrm{c}-\mathrm{b}=0.6 \mathrm{~mm}$; and $\varepsilon_{\text {rel }}=2.5$. With this choice of parameters, the highfrequency characteristic impedance of the line is $50 \Omega$. To state the difference between the dielectric loss on weak conductor and a good conductor, we consider the coaxial conductor for tellurium $\left(\sigma=5 \times 10^{3} \mathrm{~S} / \mathrm{m}\right)$ and copper $\left(\sigma=5.76 \times 10^{7} \mathrm{~S} / \mathrm{m}\right)$ both cases.

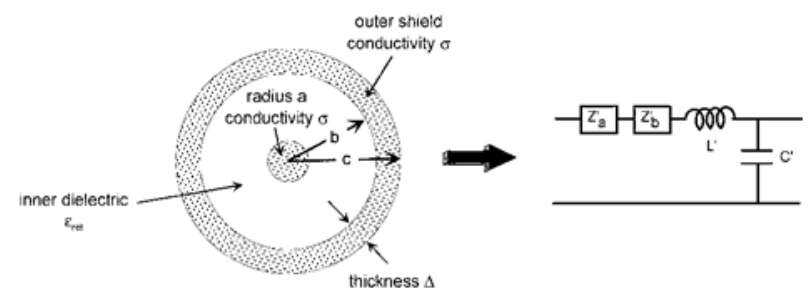

Figure 2. Coaxial transmission line and its per-unit-length equivalent circuit

In (For the transmission line, we assume that the total length of the line is $\mathrm{L}=1 \mathrm{~m}$ and that the source location is at $x_{s}=0.2$ from the left end of the line (see Fig. 1). The load impedances of the line are assumed to be resistive, with values $Z_{L_{1}}=R_{L_{L_{1}}}=100 \Omega$ and $Z_{L_{3}}=R_{L_{2}}=10 \Omega$.For a coaxial line, the per-unit length line inductance and capacitance and dc resistance and shunt conductance parameters as

$$
\begin{aligned}
& L^{\prime}=\frac{\mu_{0}}{2 \pi} \ln \left(\frac{b}{a}\right) \text { and } C^{\prime}=\frac{2 \pi \varepsilon_{0} \varepsilon_{r e l}}{\ln (b / a)}, \\
& R^{\prime}=\frac{1}{\pi \sigma}\left(\frac{1}{a}+\frac{1}{2 b \Delta}\right) \text { and } G^{\prime}=\frac{2 \pi \sigma^{\prime}}{\ln (b / a)}
\end{aligned}
$$

Where $\sigma^{\prime}$ is a leakage of the fill media conductivity, the ideal state where $\sigma^{\prime}=0$.

While the BLT solutions in (14) are expressed in terms of both a current and voltage source, only a voltage source is considered in this example. The transient waveform produced by this source is assumed to be representative of a fast pulser having a rise time of the order of $100 \mathrm{ps}$ and a fall time of about $4 \mathrm{~ns}$. A reasonable representation of such a pulse is given by the expression

$$
\begin{aligned}
& \mathrm{V}(t)=\mathrm{V}_{p}(1+\Gamma) e^{-\left(\frac{t-t_{s}}{t_{f}}\right)} \times\left\{0.5 \operatorname{erfc}\left(-\sqrt{\pi} \frac{t-t_{s}}{t_{r}}\right) \Phi\left(-\left(t-t_{s}\right)\right)\right. \\
& \left.+\left[1-0.5 \operatorname{erfc}\left(\sqrt{\pi} \frac{t-t_{s}}{t_{r}}\right)\right] \Phi\left(t-t_{s}\right)\right\}
\end{aligned}
$$

where $\operatorname{erfc}(\cdot)$ denotes the complementary error function, and $\Phi(\bullet)$ is the unit step function.4 Using this equation, it is found that the following parameters provide a good fit to the such a pulser waveform:

$\mathrm{V}_{p}=10 \mathrm{kV}$ and $\Gamma=0.024$ and $t_{r}=100 \mathrm{ps}$ and $t_{\mathrm{s}}=0.2 \mathrm{~ns}$ and $t_{f}=4 \mathrm{~ns}$, Fig. 3 illustrates this voltage waveform, which is used in the analysis to follow.

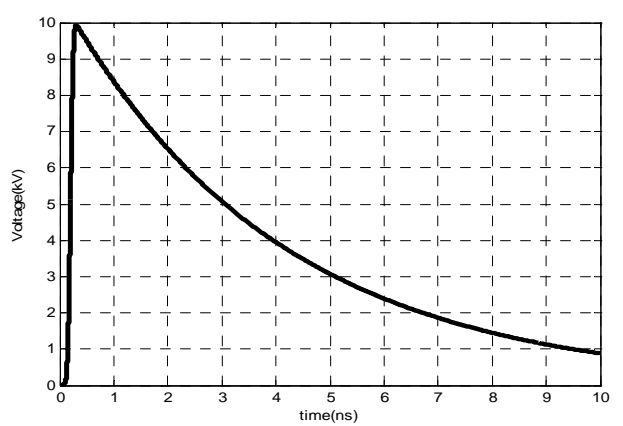

Figure 3. Voltage source waveform

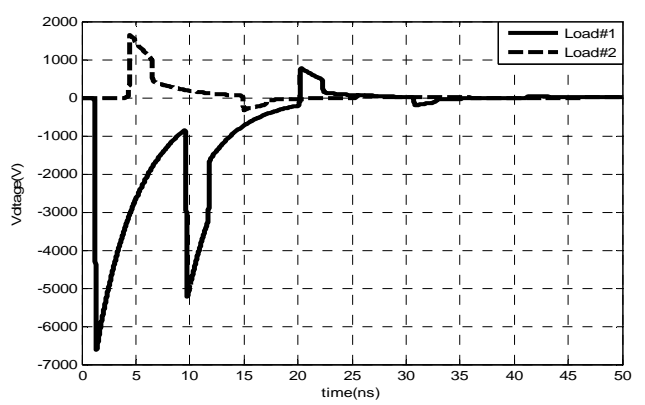

(a) $\sigma^{\prime}=0 \quad S / m$

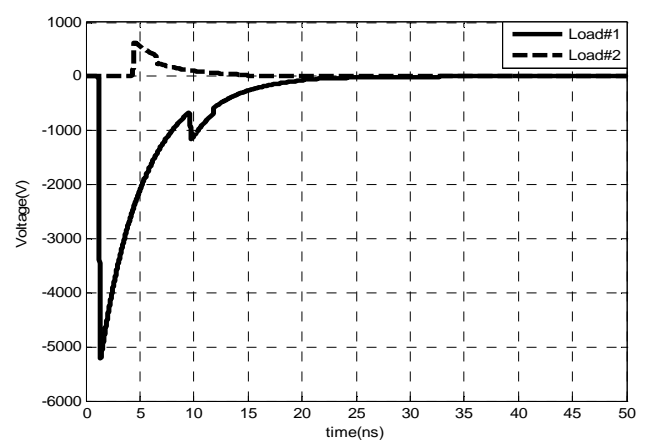

(b) $\sigma^{\prime}=5 \times 10^{-3} \mathrm{~S} / \mathrm{m}$

Figure 4. Good conductor (copper) coaxially load voltage. (a) No dielectric loss; (b) dielectric loss

Using the transient-BLT equations (14) for the load voltages, a direct time-domain solution for the coaxial transmission line load voltages has been obtained in Fig. 4. 
The Fig.4 and Fig.5 illustrates the dielectric loss does coaxial terminal load response voltage generated impact load response voltage than lossless processed produce attenuation in the case of the presence of the dielectric loss.

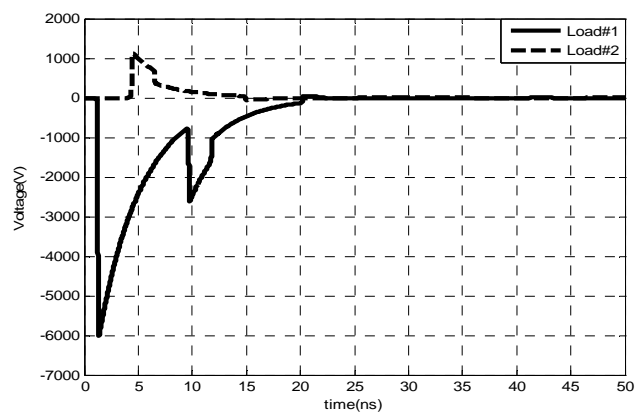

(a) $\sigma^{\prime}=0 \quad \mathrm{~S} / \mathrm{m}$

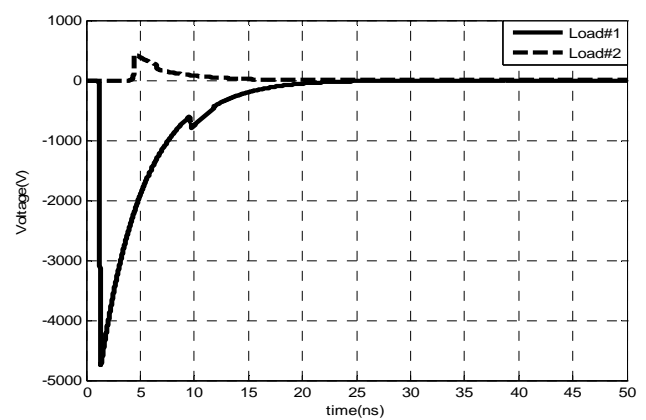

(b) $\sigma^{\prime}=5 \times 10^{-3} \mathrm{~S} / \mathrm{m}$

Figure 5. Weak conductor (tellurium) coaxial load voltage (a) No dielectric loss; (b) dielectric loss

The Fig.6 illustrates as the drain conductivity increases, the voltage of the load \# 1 presents nonlinear attenuation, and can see the load response of the voltage decay in the early ( $t=10 \mathrm{~ns}$ ) good conductor (copper) coaxially is relatively fast; voltage attenuation of substantially the same speed and in the load response of the late $(t=15 \mathrm{~ns})$ good conductor (copper) and weak conductor (tellurium) coaxial.

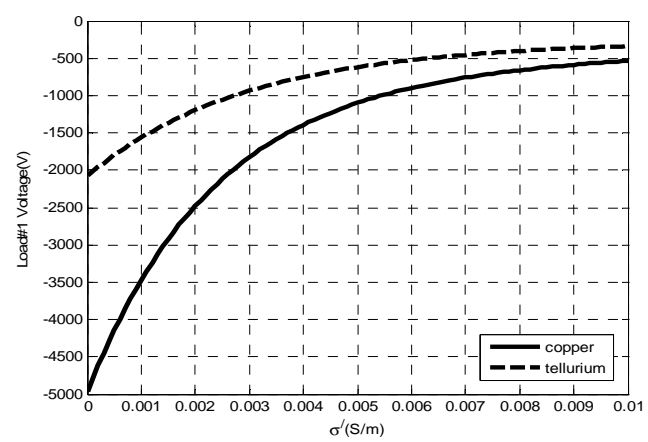

(a) $t=10 \mathrm{~ns}$

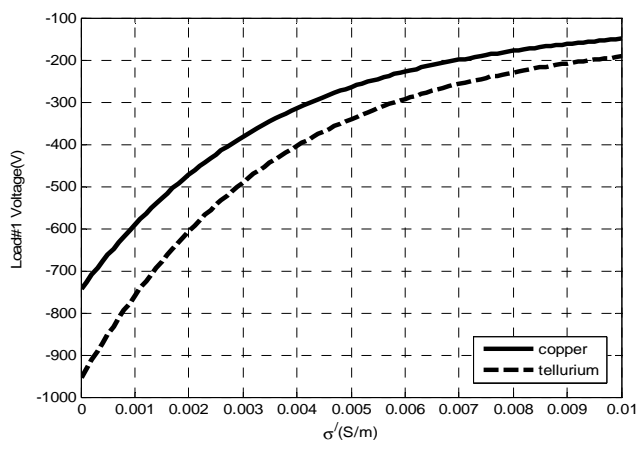

(b) $t=15 n s$

Figure 6 . The coaxially load \# 1 voltage electrical conductivity changes with the drain

\section{OBSERVATIONS}

By the above theoretical analysis and numerical simulation, we know that due to the dielectric loss caused by the leakage of the medium so that the response voltage of the coaxial transmission line terminal load attenuation, and this attenuation is nonlinear. Good conductor of coaxial transmission line, the signal attenuation in the initial relatively rapid, while the rate of decay in the late signal is substantially the same, the little difference between a good conductor with a weak conductor. This requires that we better insulation properties of the medium, in the actual design of the coaxial transmission line is particularly important, especially for a good conductor of coaxial transmission line dissemination of high-frequency signals, to avoid the dielectric loss of the signal propagating to ensure the dissemination of signals authenticity. Although this example is based on the coaxial transmission line, but the corresponding conclusions can be extended to a uniform transmission line, uniform transmission line system design has practical significance.

\section{REFERENCES}

[1] F. M. Tesche, "Development and use of the BLT equation in the time domain," [J]. IEEE Trans. Electromagn. Compat., vol. 49, no. 1, pp. $3-11$, Feb. 2007

[2] F. M. Tesche, "On the analysis of a transmission line with nonlinear terminations using the time dependent BLT equation," [J]. IEEE Trans. Electromagn. Compat., vol. 49, no. 2, may. 2007.

[3] F. M.Tesche, EMC Analysis Methods and Computational Models [M].New York: Wiley, 1997.

[4] C. E. Baum, "Extension of the BLT equation into time domain," [J]. in AFRL EMP Interaction Note 553, Kirtland AFB, New Menico, Mar. 24, 1999.

[5] F. M. Tesche and C. M. Butler, "On the addition of EM field propagation and coupling effects in the BLT equation," [J]. Kirtland AFB, Albuquerque,NM,

[6] K.S.H. Lee. EMP Interaction: Principles, Techniques and Reference Data. [M]. New York: Taylor and Francis, 1989. 